\title{
Seaborgium's complex studies
}

\author{
Christoph E. Düllmann reflects on the excitement, and implications, of probing the reactivity of heavy \\ element seaborgium.
}

T he periodic table of elements devised by Mendeleev well over a hundred years ago has become a prime tool for chemists, as well as a widely recognized symbol of chemistry - such that it is easy to forget it remains a live document. The addition of a new element every now and then stirs up much interest, in particular with regards to whether its properties conform to the table's established structure and trends.

This isn't necessarily a given, especially for heavy elements. Indeed, the many protons inside the nucleus accelerate proximal electrons to a substantial fraction of the speed of light, causing the electrons' mass to increase owing to relativistic effects, and their orbits to deviate from the hypothetical ones they would otherwise follow. This is hardly noticeable in lighter elements, but the transactinide series, with atomic numbers $\geq 104$, makes for an ideal 'laboratory' for studying these effects.

Studies are hindered, however, by the small quantities and short lifetimes of these elements, generally single atoms existing for seconds or minutes at most. Still, elaborate automated set-ups for performing gaschromatography volatility measurements of single atoms or molecules have enabled studies on compounds of all elements up to hassium, and recently also on copernicium and flerovium (elements 108, 112 and 114, respectively) ${ }^{1}$. Comparisons with analogues of lighter homologues in their respective groups allowed conclusions to be drawn about the validity of trends established in the periodic table.

Element 106 - named seaborgium (Sg) after Glenn T. Seaborg (pictured) - was discovered in 1974. The first chemical studies, of $\mathrm{SgO}_{2} \mathrm{Cl}_{2}$, showed its volatility conformed to the trend established in group 6 by $\mathrm{MoO}_{2} \mathrm{Cl}_{2}$ and $\mathrm{WO}_{2} \mathrm{Cl}_{2}$, in agreement with relativistic quantum chemical calculations. These results, together with later studies on the oxide-hydroxide system and two liquid-phase

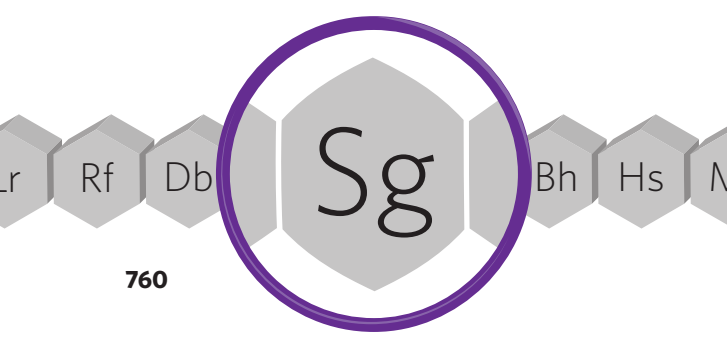

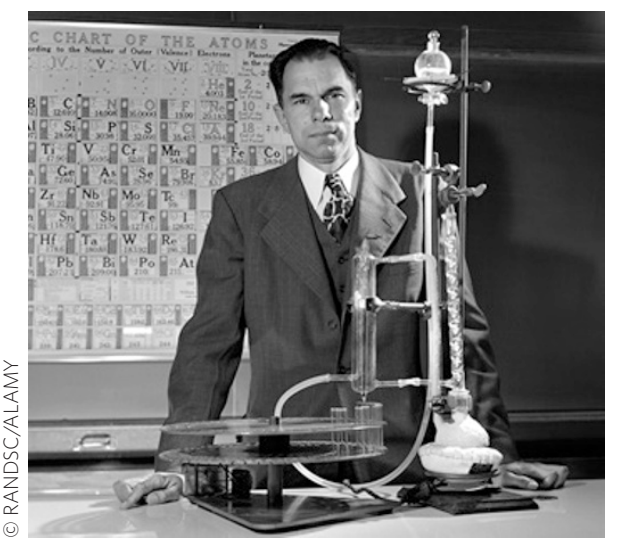

experiments, constitute our current knowledge on seaborgium's chemistry and establish it as a full member of its group.

Despite these successes, compounds featuring transactinide atoms have remained poorly available. Atoms of transactinides are formed in fusion reactions of lighter atomic nuclei using intense high-energy ion beams and are thus often only available for study in an environment hostile to most chemical systems: a beam-induced plasma.

Our efforts to overcome this technical limitation started about 10 years ago, and have recently shown some success: seaborgium ions have been separated from the ion beam in a magnetic field ${ }^{2}$, then collected in a gasvolume at ambient conditions, enabling the synthesis of seaborgium hexacarbonyl - the first transactinide complex in which the metal is in a reduced oxidation state. This technique was pioneered with zirconium and hafnium ${ }^{3}$, and the focus was subsequently set on group 6 hexacarbonyl complexes.

Experiments worked marvellously with molybdenum and tungsten ions stopped in CO-containing gas ${ }^{4}$. We teamed up with researchers from RIKEN, Wako-shi, Japan, who had perfected seaborgium's synthesis and separation. In over two weeks of aroundthe-clock experimenting, our detection system registered the decay of 18 atoms of seaborgium and its decay products - a high-statistics experiment by transactinide chemists' standards. Passing the volatile $S g$ species over progressively colder surfaces allowed us to measure the temperature at which they immobilize - owing to physisorption, as our data show. The deduced adsorption enthalpy is similar to those of $\mathrm{Mo}(\mathrm{CO})_{6}$ and $\mathrm{W}(\mathrm{CO})_{6}$, corroborating our assignment ${ }^{5}$ of the species to $\mathrm{Sg}(\mathrm{CO})_{6}$ as also supported by theoretical work ${ }^{6}$.

Fascinating perspectives for further studies arise: syntheses of other complexes featuring a metal-carbon bond appear feasible, paving the way towards transactinide organometallic compounds. $\mathrm{Sg}(\mathrm{CO})_{6}$ currently represents the heaviest element carbonyl, adding to the riches of carbonyl chemistry over a hundred years after the discovery of a carbon monoxide complex ${ }^{7}, \mathrm{Ni}(\mathrm{CO})_{4}$. After the $\mathrm{Sg}(\mathrm{CO})_{6}$ identification, a next step may include measuring the seaborgium-carbon bond strength, for which theoretical data are available ${ }^{8}$. Not least, carbonyl complexes of yet heavier elements appear in reach as well.

Forty years after seaborgium's discovery, twenty years after its first chemical study, a hexacarbonyl compound with seaborgium in a reduced oxidation state has been synthesized and will be scrutinized. We feel honoured to continue Glenn Seaborg's legacy by expanding transactinide chemical studies to new systems, and measuring previously inaccessible characteristics. $\mathrm{Sg}(\mathrm{CO})_{6}$ is an exciting step on this path.

\section{CHRISTOPH E. DÜLLMANN is at the} Johannes Gutenberg University and the Helmholtz Institute, 55099 Mainz, Germany; and at the GSI Helmholtz Centre for Heavy Ion Research, 64291 Darmstadt, Germany. e-mail: c.e.duellmann@gsi.de

\footnotetext{
References

1. Türler, A. \& Pershina, V. Chem. Rev. 113, 1237-1312 (2013).

2. Düllmann, Ch. E. Eur. Phys. J. D 45, 75-80 (2007).

. Düllmann, Ch. E. et al. Radiochim. Acta 97, 403-418 (2009)

4. Even, J. et al. Inorg. Chem. 51, 6431-6433 (2012).

5. Even, J. et al. Science 345, 1491-1493 (2014).

6. Pershina, V. \& Anton, J. J. Chem. Phys. 138, 174301 (2013).

7. Werner, H. Angew. Chem. Int. Ed. 29, 1077-1089 (1990).

8. Nash, C. S. \& Bursten, B. E. J. Am. Chem. Soc. 121, 10830-10831 (1999).
} 